\title{
Global visual completion of quasi-regular shapes
}

Tessa C J de Wit, Rob J van Lier

Nijmegen Institute for Cognition and Information (NICl), University of Nijmegen, PO Box 9104, NL 6500 HE Nijmegen, The Netherlands; e-mail: t.dewit@nici.kun.nl

Received 26 November 2001, in revised form 22 March 2002; published online 11 July 2002

\begin{abstract}
The topic of amodal completion has often been investigated by using partly occluded shapes that are regular. In research that has typically been done with displays such as these regular shapes, it has been shown that global aspects of a shape can determine completion. To see how robust these global influences in the completion process are, we investigated quasiregular shapes, ie shapes with a certain overall regularity but not based on metrical identities. First, in experiment 1 participants had to complete quasi-regular shapes in a drawing task. Then, in experiment 2 the primed-matching paradigm was used. Results from both experiments provided evidence for global completions. In experiment 3 we found that multiple global completions can be primed, which, as a control experiment showed, cannot be explained by some inability of the visual system to see the difference between the different completions. These data support the notion that global influences on visual occlusion are apparent even when the partly occluded stimulus is outside the domain of regular shapes. Implications for a global approach are provided.
\end{abstract}

\section{Introduction}

In the world that surrounds us, most objects are partly hidden from our view by other objects. In some way our brain nevertheless makes sense of the still available information from the visible part of the partly occluded object, so that we do experience these objects as whole objects. Various theories exist about the way the brain accomplishes this visual completion. Roughly speaking, these theories can be categorised into three main groups: local theories, global theories, and integrated theories.

The local theories derive from the Gestalt law of good continuation, which states that any curve will proceed in its own natural way (Wertheimer 1923). In other words, a line will continue in the same direction (Koffka 1962). For instance, the shape on the left in figure la is partly occluded, which in figure $1 \mathrm{~b}$ is completed in a way that local theories would predict. Kanizsa $(1975,1979$, 1985) was an important advocate of this local account, pointing at the role of T-junctions as an index of this local completion process. Various theories formalised this law of good continuation, such as the theory of visual interpolation by Kellman and Shipley (1991). These authors predicted smooth curve completion if the angle between the virtual linear extensions of the occluded shape is equal or greater than $90^{\circ}$. This principle was referred to as the reliability criterion. Boselie and Wouterlood (1992) criticised this principle on several grounds and proposed their own local model (Wouterlood and Boselie 1992). In their good-continuation model, it is assumed that there is a tendency to describe a pattern

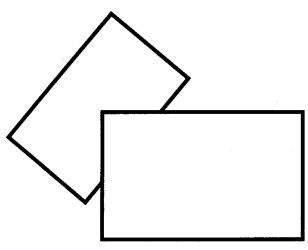

(a)

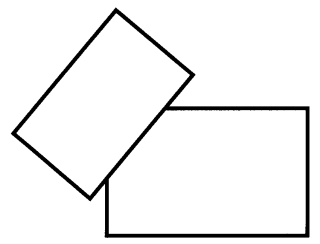

(b)
Figure 1. (a) The shape on the left is partly occluded by the shape on the right. (b) The completion of the left shape as predicted by local and global theories. 
by the smallest number of contour elements and changes of direction within and between these contour elements. Although this is a local model, Wouterlood and Boselie (1992) do acknowledge influences from regularities in the shapes. But to rule out these regularitybased influences, they restricted the stimulus domain to which their model applies to irregular shapes (see also Boselie 1994).

The global theories, on the other hand, have their roots in the law of Prägnanz, as introduced by Koffka (1962). This law states that perceptual organisation will always be as 'good' as the dominating conditions allow. Although the term 'good' is not specified in this definition, one may think of properties such as symmetry and repetition. Generally it can be said that the information from the total visible configuration of the partly occluded object is used in the completion process to give rise to a complete object that is as simple as possible to describe. The completion of figure $1 \mathrm{~b}$ also shows the global completion of the occluded shape in figure 1a. So for this particular shape, local and global theories predict the same completion. In figure $2 \mathrm{a}$ an occluded shape is shown for which local and global theories would predict different completions, and in figure $2 \mathrm{~b}$ the local completion is depicted. Note that the specific predictions of the various approaches might differ from each other. The given local completion in figure $2 \mathrm{~b}$ is based on linear good continuation and agrees, for example, with the model proposed by Wouterlood and Boselie (1992). [Note that the approach of Kellman and Shipley (1991) would not predict completion here because the edges do not meet the relatability criterion; see also Boselie and Wouterlood (1992).]
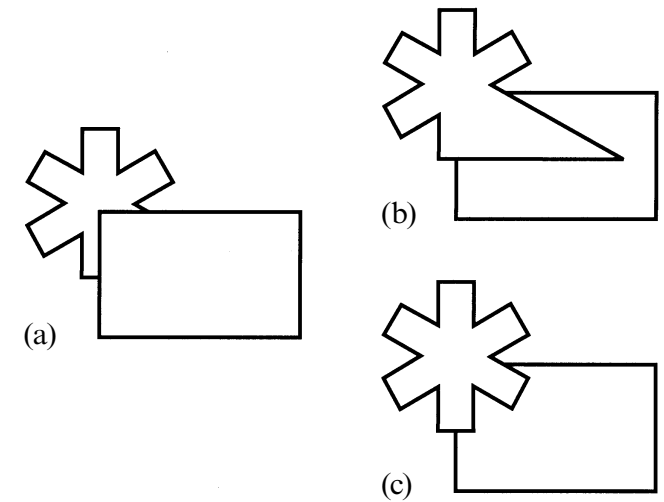

Figure 2. (a) The shape on the left is partly occluded by the shape on the right. (b) Completion of the left shape as predicted by some local theories. (c) Completion of the left shape as predicted by some global theories.

As mentioned, global completion is characterised by the overall appearance of goodness of the shape, as depicted in figure 2c. Hochberg and McAlister (1953) tried to make the goodness idea more concrete through the global-minimum principle. This principle states that a figure will be interpreted such that the information that defines it is minimal. Leeuwenberg $(1969,1971)$ formalised this global-minimum principle by means of a descriptive system, the structural information theory, and in their turn, Buffart et al $(1981,1983)$ applied it to occlusion phenomena. The global-minimum principle seemed to work for a lot of stimuli, but, as for example Rock (1983), Kanizsa (1985), and Boselie (1988) showed, the simplest code does not always predict the preferred completion of an occluded figure. For some time, evidence seemed to point in the direction of mainly local completions. However, Sekuler (1994) and Sekuler et al (1994) showed that global processes could also dominate the completion of occluded figures, a finding that was also reported by van Lier et al (1995a), and van Lier and Wagemans (1999). 
These kinds of results suggested that there are both local and global processes that influence the way occluded shapes are completed. Different integrative models have been put forward to disentangle these influences. Sekuler (1994) proposed a qualitative integrative model in which both local and global influences play a role in the amodal completion process. The model predicts different completions on the basis of factors such as good continuation, symmetry, repetition, familiarity, and context. Another integrative model was put forward by van Lier et al $(1994,1995 b)$ in which the nature of a completion is predicted on the basis of a quantification of both global and local aspects. By calculating the complexity of these global and local aspects, the completion with the lowest overall complexity is predicted.

One drawback of the research that has been done so far is that the stimulus domain of most experiments was fairly restricted. Although in some experiments less regular shapes were used (see eg Shipley and Kellman 1992; Yin et al 1997; Rubin 2001), the fact is that most shapes and objects that we can see in our world are not perfectly regular. Obviously, local theories would predict a local completion for these partly occluded nonregular shapes. The global theories and the integrated theories, so far, will also predict local influences to dominate in the completion process of these shapes. The reason that these theories would not predict global influences to dominate in these shapes lies in the absence of overall regularity based on metrical identities in the visible part of the partly occluded form (see figure 3a). However, regularities that are not formed by metrical identities may still influence the way such a partly occluded form is completed (van Lier 1999). For example, the occluded shape in figure $3 \mathrm{a}$ is not a perfectly regular shape in the strict metrical sense. There is regularity in the shape though, which can be found in the nature of the protrusions. We will refer to these kinds of non-metrically regular shapes as quasi-regular shapes. Comparing the local (figure $3 \mathrm{~b}$ ) and the global completion (figure 3c), intuitively shows that a global completion seems more plausible. In line with this point, we argue here that global influences can still be found in the completion process of partly occluded shapes that lie outside the usual, regular stimulus domain. Of course, because of the lack of a perfect overall regularity it is not possible to make a prediction for one specific global completion.
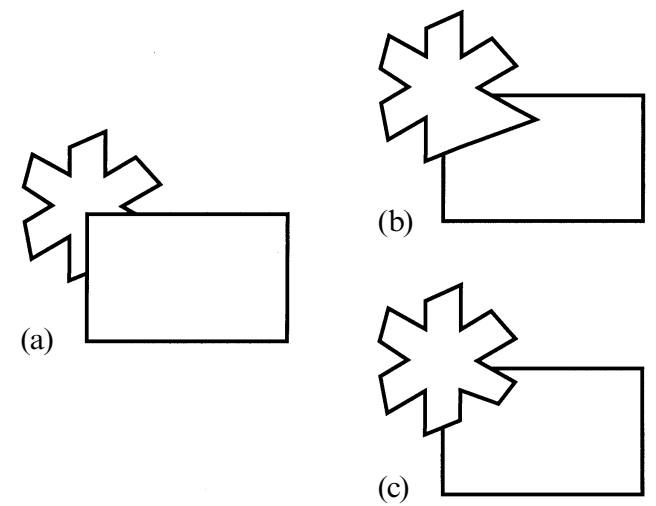

Figure 3. (a) The quasi-regular shape on the left is partly occluded by the shape on the right. (b) Completion of the left shape as predicted by some local theories. (c) A possible global completion.

To investigate the presence of global influences in the completion process for partly occluded shapes outside the domain of perfectly regular shapes we expanded the usual stimulus domain to quasi-regular shapes. We first investigated this issue using a drawing task (as in Buffart et al 1981; Boselie 1988) to see how people would spontaneously complete such a shape, if they are not restricted in their responses by a set of completions made beforehand. Later on we also used the primed-matching paradigm, to investigate the question in a more controlled experiment. 


\section{Experiment 1 (pilot experiment)}

\subsection{Method}

2.1.1 Participants. A class of nineteen students at the University of Nijmegen participated in this experiment. All had normal or corrected-to-normal vision.

2.1.2 Stimuli. Three sets of stimuli were constructed by superimposing different contour sequences (alternating lines and angles) on a virtual circle (figure 4). The shapes were composed of different sequences of concave and convex angles. The contour sequence of the first set had one concave and one convex angle alternating with lines having about the same length (figure 4a). The second set consisted of two subsequent concave angles and one convex angle alternating with differing line lengths (figure $4 \mathrm{~b}$ ), and the third set consisted of a pair of two concave angles and two convex angles alternating with similar line lengths (figure 4c).

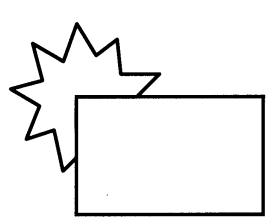

(a)

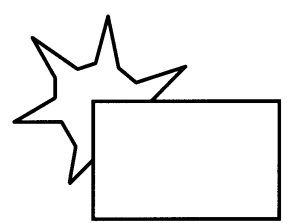

(b)

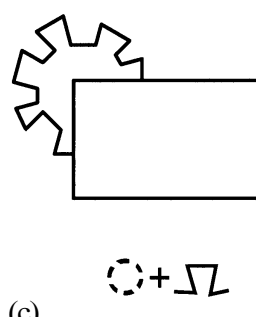

(c)

Figure 4. The three different sets for experiment 1: (a) set 1; (b) set 2; (c) set 3. Below each shape a virtual circle is depicted together with the superimposed pattern of lines and angles placed by means of which the quasi-regular shapes can be composed.

Every set had three different variations; the difference being in the number of visible protrusions (which was either 4,5 , and 6 for set 1; 5, 6, and 7 for set 2; while for set 3 this was 6,7 , and 8 ), so there was a total of nine stimuli. The shape of interest was always occluded by a rectangle that was $3.9 \mathrm{~cm}$ by $2.4 \mathrm{~cm}$. The horizontal and vertical extents of the figures were about $5.3 \mathrm{~cm}$ by $3.9 \mathrm{~cm}$. Stimuli were presented on nine separate sheets of paper, with black line drawings on a white background.

2.1.3 Procedure. Participants received the nine sheets of paper in a randomised order, with the only constraint that shapes belonging to the same set never followed each other. The sheets of paper were placed on a table at a normal viewing distance. Participants were instructed to decide whether they perceived the two visible figures as being a mosaic (two figures juxtaposed to each other) or as a shape partly occluded by a rectangle. Only if participants perceived it as a partly occluded shape, they had to draw the occluded part in the picture. The mosaic option was added to give participants the freedom to perceive the pictures in an alternative way. There was no time limit for the task.

\subsection{Results}

The responses of the participants were classified into the following categories: global, local, mosaic, and a rest category. The global category referred to drawn completions that followed the pattern of alternating lines and angles that was already present in the visible part of the partly occluded shape. Also, the sizes of the protrusions in the completion were roughly similar to those of the visible part of the partly occluded shape. The local completions were drawings where the lines that continued behind the occluded rectangle were simply extended to the point where both lines crossed each other. For the first and second set, this resulted in one continuous line, and for the third set this resulted in two lines and an extra angle. One of the 171 response sheets was not returned, and 15 were left blank, and have been classified as 'mosaic'. 
Of the remaining 155 drawings, most drawings, 147 - ie $86 \%$ of the total amount of drawings - were classified as global, one completion was classified as a local completion, and seven drawings $(4.1 \%)$ as belonging to the rest category. The rest-category drawings consisted of alternating angles and lines, but did not follow the overall pattern of alternating angles and lines. These drawings were only found for shapes of set 2. The number of protrusions did not appear to have an effect on the way the shapes were completed.

\subsection{Discussion}

Most participants tend to complete partly occluded quasi-regular shapes in a global way, by continuing the pattern of the visible part in the completion. Only one completion was classified as local, and there were a couple of exceptions where participants completed the partly occluded shape in an ambiguous way, ie completions with protrusions that did not follow the overall pattern of alternating lines and angles. These completions, however, were more similar to global completions than to local completions.

The drawing method that was used here has the advantage of the aforementioned freedom of the participant to complete the partly occluded form in any preferred way. For this reason, various researchers already used this method to investigate the domain of visual completions (eg Buffart et al 1981; Boselie 1988). Although data clearly show that quasi-regular forms are completed in a global way, we have to be cautious. Since participants have quite some time to think about the completions, this consequently makes this method highly susceptible to the influence of higher cognitive inferences. Therefore, a more sophisticated paradigm was needed to look at the perceptual relevance of amodal completion of partly occluded quasi-regular shapes.

\section{Experiment 2}

The primed-matching paradigm provided us with a good tool to investigate the role of global factors on amodal completion (Beller 1971; Sekuler and Palmer 1992). The rationale behind the primed-matching paradigm is that showing a prime before an ordinary matching task improves the participant's performance on this task if there is a high similarity between the prime and the test shapes. In the matching task a participant has to make a judgment about the similarity of a pair of shapes (ie the test pair) that are presented simultaneously. Showing a prime before the presentation of the test pair speeds up the reaction time (RT) for 'matching' test pairs the shapes of which are representationally similar to the prime (Beller 1971). In the context of amodal completion, this paradigm can be applied by using a partly occluded shape as a prime. This so-called occlusion prime is expected to speed up the RT for 'matching' test pairs of which the shapes are congruent with the completed form of the occluded shape in the prime (Sekuler and Palmer 1992). By comparing the effect of an occlusion prime on differently perceptually completed test pairs, it can be tested which completion is formed. Namely, the test pair with the shapes that are most similar to the completed form of the partly occluded shape will be the test pair of which the RT speeds up most after the occlusion prime. Sekuler and Palmer (1992) applied this paradigm to investigate the time course of the interpretation of partly occluded shapes, contrasting mosaic interpretations with amodal completions. They found that, if occlusion primes were presented for $200-400 \mathrm{~ms}$, the effect of the occlusion prime was similar to the effect of an amodally complete prime. From this, they concluded that completions take a certain time before a completion interpretation of a partly occluded shape is formed.

Here, we are interested in the type of completion that is formed after seeing a partly occluded quasi-regular shape. To be able to see if the completions of quasi-regular shapes are global or local, effects of the occlusion primes are compared to effects of primes that are constructed to be the global and local completions of the occluded shape in 
the occlusion prime. These primes are called the foreground primes. If there are global influences in the completion process of quasi-regular shapes, we expect the effect of the occlusion primes to be similar to the effect of the global foreground primes, but not similar to the effects of the local foreground primes. In other words, we expect the occlusion prime to have the largest prime effect on the global test pairs.

\subsection{Method}

3.1.1 Participants. Twenty-two students at the University of Nijmegen participated in this experiment. All had normal or corrected-to-normal vision. Participants received either a payment or course credit.

3.1.2 Stimuli. The stimuli comprised the same three sets of figures that were used in experiment 1 (figure 4), but now only one exemplar of each set was used, which was always the middle form in terms of number of protrusions. For each set, four different primes were constructed: an occlusion prime (as shown in figure 4a), a global (foreground) prime (see figure $5 \mathrm{a}$ for the global prime of the first set), a local (foreground) prime (figure 5b), and an anomalous (foreground) prime (figure 5c). The anomalous prime was added to the stimulus set to create a control condition that was a theoretically possible but highly unlikely completion. The anomalous prime was constructed by changing the pattern of alternating angles or by greatly increasing the line lengths in the completed part. To be able to determine the amount of speed-up for each prime, a no-prime condition was used as well. The no-prime display simply consisted of a dot in the middle of the screen.

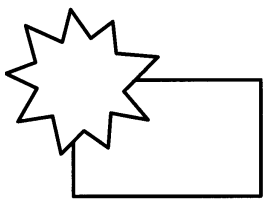

(a)

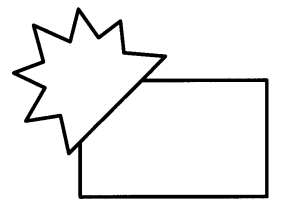

(b)

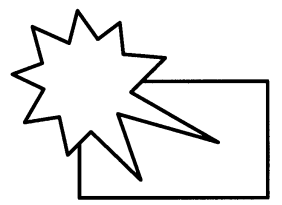

(c)

Figure 5. The three possible foreground primes for the occluded shape of set 1 of experiment 2: (a) global prime; (b) local prime; (c) anomalous prime.

A control set was added to the stimulus set. This control set comprised regular shapes and was put in to check whether we observed the same basic effects as previous researchers investigating amodal completion (eg Sekuler and Palmer 1992). This set had a partly occluded circle, of which global and local completion strategies would give rise to the same complete circle. For this set, we also used an anomalous completion.

The test display was formed by placing two completed shapes on each side of the original prime; for an example of this display see figure 6. This arrangement was chosen to prevent the prime from masking the test pair (similar to Sekuler and Palmer 1992). Also, during this test phase, the shape on the right in the prime (ie the rectangle that is irrelevant for the test stimulus) was positioned at the bottom of the display. This was done to prevent an apparent movement and accompanying shape change that may deteriorate the prime effect (Sekuler and Palmer 1992). The test pairs of each set always consisted of either a matching pair (two completely identical shapes, as in figure 6), or a non-matching pair that was made up of a combination of the different possible completions of one set. Here, the left-right position was varied for every pair, so each quasi-regular set had three matching (global-global, local-local, and anomalous-anomalous) and six non-matching test pairs (global-local, local-global, global-anomalous, anomalous-global, local-anomalous, and anomalous-local). In the fourth set there were two matching and two non-matching pairs, adding up to a total number of 31 different test pairs. The number of matching test pairs was presented as many times in the experiment as the number of non-matching test pairs, and the 


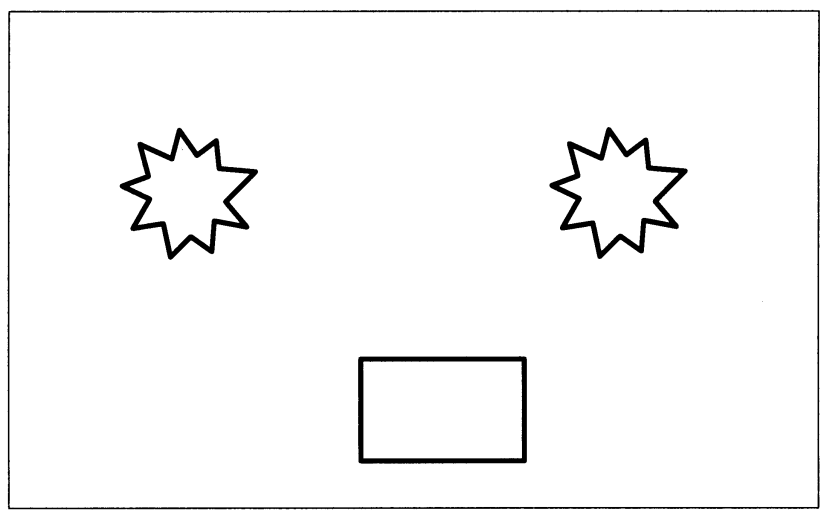

Figure 6. An example of a display containing a matching test pair. The rectangle at the bottom was presented for control purposes (see text).

number of presentations was also equal for every kind of completion. Practice stimuli were created that consisted of simple shapes that were not used in the actual experiment.

The maximum horizontal and vertical extents of the figures in the prime display were $1.7 \mathrm{deg}(3.5 \mathrm{~cm})$ and $1.4 \mathrm{deg}(3 \mathrm{~cm})$; the maximum horizontal and vertical extents of the figures in the test display were $4.8 \mathrm{deg}(10 \mathrm{~cm})$ and $3.1 \mathrm{deg}(6.5 \mathrm{~cm})$. All figures were black on a white background.

3.1.3 Procedure. The experiment was conducted in a dimly lit room, where participants sat approximately $120 \mathrm{~cm}$ from the screen with their heads stabilised by a chin rest. First, a cross appeared in the middle of the screen for fixation purposes for $500 \mathrm{~ms}$; after that, the screen went blank for $50 \mathrm{~ms}$, followed by the prime that was shown for $500 \mathrm{~ms}$. Then, after a $17 \mathrm{~ms}$ interstimulus interval, the test pair appeared. The test stimuli were always of the same set as the prime, and remained on the screen until the participant responded. The trials were presented continuously, but participants could take a pause between the trials by pressing a button. Participants responded by pressing one button for matching test pairs and another button for non-matching pairs of test stimuli. Half of the participants used their dominant hand for "same" responses, while the other half used their non-dominant hand for "same" responses. Responses were measured to the nearest millisecond. The experiment was run with SuperLab Pro, version 2.0 (Cedrus Corporation). The order of the presentation was randomised for each participant.

Similar to Sekuler and Palmer (1992), participants were instructed to pay attention to the left part of the prime to encourage attending to the prime. Also, participants were instructed to respond as accurately and fast as possible. Before the 352 experimental trials, participants received 16 practice trials, with simple, geometric figures, different from the ones used in the experimental trials. To get participants to respond as fast as possible, they received feedback on their speed in the practice trials.

\subsection{Results}

Like Sekuler and Palmer (1992), we analysed correctly answered matching test pairs (94.5\% of all trials), because prime effects only seem to occur for identical test pairs (Beller 1971). We defined the effect of a prime as the difference between the reaction time of a prime trial and the reaction time of a no-prime trial:

$$
\mathrm{PE}(\mathrm{TP} \mid \mathrm{P})=\mathrm{RT}(\mathrm{TP} \mid \mathrm{NP})-\mathrm{RT}(\mathrm{TP} \mid \mathrm{P}),
$$

where PE is the prime effect, RT the reaction time, TP any test pair, NP stands for no prime, and $\mathrm{P}$ stands for any prime. First, the results obtained from the control set 
were analysed to check whether we observed the same basic effects as Sekuler and Palmer (1992). A paired-samples $t$-test was performed to see how the RTs for the test stimuli were affected by the different primes. RTs for test pairs were shortened by 'congruent' foreground primes, foreground primes that were completed in the same way as the shapes in the test pair [PE (circle $\mid$ circle): $t=3.627, p<0.005$; PE (anomalous|anomalous): $t=-2.899, p<0.01]$. The occlusion prime facilitated the test pair with the two circles [PE (circle|occlusion): $t=-2.357, p<0.05$ ], but not the test pair with the two anomalous completions [PE (circle|anomalous): $t=0.822, p=0.42$ ]. All these findings were as expected, so the paradigm seemed like a valid way to investigate the completion process. The control set was not used in any of the further analyses.

In figure 7, the mean prime effect is plotted for the foreground and occlusion primes as a function of different test pairs. A repeated-measures ANOVA was performed for prime effect with stimulus set (3), type of prime (4), and test pair (3) as independent variables and $\mathrm{PE}$ as the dependent variable. This revealed a main effect for test pair $\left(F_{2,20}=7.80, p<0.005\right)$ and a significant prime $\times$ test pair interaction $\left(F_{6,16}=11.22\right.$, $p<0.001)$. A paired-samples $t$-test was performed to see if the RTs in the congruent prime conditions were significantly different from the RTs in the no-prime conditions for the three quasi-regular sets of stimuli. Overall, for the three sets it was found that primes shortened the RT for the congruent test pair [PE (global|global): $t=-7.350$, $p<0.001$; PE (local|local): $t=-3.145, \quad p<0.005 ; \quad$ PE (anomalous|anomalous): $t=-6.497, p<0.001]$. More importantly, the occlusion prime significantly facilitated the comparison of the global test pair [PE (global|occlusion): $t=-5.850, p<0.001$ ], but not of the other test pairs [PE (local|occlusion): $t=1.347, p=0.192$; and PE (anomalous|occlusion): $t=0.406, p=0.689]$. Tests were also performed on the three sets separately, the results of which can be found in table 1 .

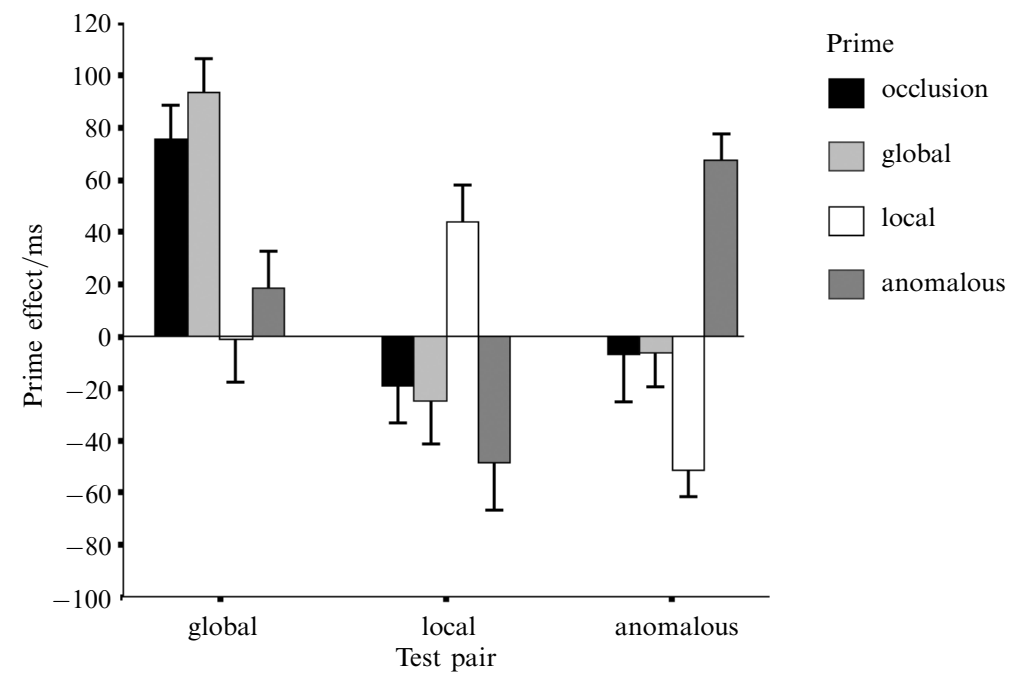

Figure 7. Mean prime-effect as a function of different test pairs for experiment 2. The bars represent mean standard error.

\subsection{Discussion}

The results from the control set (the regular shapes) indicate that the paradigm functioned as it should. That is, we got the same basic results as Sekuler and Palmer (1992) in that the occluded circle primed only the expected circular completion. Also not surprisingly, performance for all test pairs was facilitated by their congruent primes, but not the other primes. These findings were found for all sets together and 
Table 1. Summary for the paired $t$-test performed for the prime effect of primes for different test pairs (1, 2, and 3). Congruent foreground primes (FP) or occlusion primes (OP), per stimulus set. Significant results are marked with an asterisk.

\begin{tabular}{lllll}
\hline Set & Primes & \multicolumn{2}{l}{ Test pair } & \\
\cline { 3 - 5 } & & global & local & anomalous \\
\hline 1 & FP & $p<0.001^{*}$ & $p<0.05^{*}$ & $p<0.05^{*}$ \\
& OP & $p<0.001^{*}$ & $p=0.789$ & $p=0.171$ \\
2 & FP & $p<0.05^{*}$ & $p=0.500$ & $p<0.001^{*}$ \\
& OP & $p<0.005^{*}$ & $p=0.207$ & $p=0.826$ \\
3 & FP & $p<0.001^{*}$ & $p<0.010^{*}$ & $p<0.001^{*}$ \\
& OP & $p<0.05^{*}$ & $p=0.139$ & $p=0.773$
\end{tabular}

for each set separately, with the only exception that the local prime of the second set did not significantly speed up RT for the local test pair. The most important finding, in the light of our question, was that the occlusion prime did speed up RT for the global test pairs, but not for the local and the anomalous test pairs. Again, this was true for all sets together and each set separately. This prime effect of the quasi-regular, partly occluded shapes in the primes for global completions provides a strong indication that global completions are formed after seeing such a partly occluded shape. Thus, global completions do occur even outside the domain of regular shapes.

This evidence for global completions of partly occluded quasi-regular shapes brought up an issue that was already briefly mentioned in the Introduction. It seems reasonable that there is not one specific global completion if there is no perfect regularity in the visible part of the partly occluded shape. Obviously, it would be quite a coincidence if the 'global' completion that we used in our experiment was exactly the completion that was formed by the participants' visual system. We expected a class of completed shapes to be susceptible to a prime effect of the occlusion prime. One can wonder about the nature of this range of plausible completions, in other words the flexibility of the visual system in amodal completion. A hint about this nature might come from a result in experiment 2 that concerns the prime effect of the occlusion prime for the anomalous test pair belonging to the first set. Even though the completion for this test pair still continues the pattern of alternating angles, as in the global completion, no prime effect was found. The difference in the prime effect must be caused by the difference from the 'global' test pair, which is in the length (and angles) of the protrusions in the completed part. For the anomalous test pair, these are much longer than the protrusions of the visible part of the occluded shape, and consequently the angle sizes differ. This indicates that the simple continuation of the pattern of alternating convex and concave angles in the visible part is not sufficient to prime that particular completion. The plausible completions of the occluded part might vary over a range of different lengths of the protrusions. We examined this possibility in a third experiment.

\section{Experiment 3}

For quasi-regular shapes, it seems plausible that there is some variability in the possible completions. To get an indication of the range of these completions that can still be primed by the occlusion prime, the lengths of the protrusions of the completed part of the stimulus set 1 from experiment 2 were manipulated. We preserved the pattern of alternating angles. Again, we used the primed-matching paradigm. If there is a wider range of possible completions, we expected the occlusion prime to speed up RTs for more test pairs than just the test pair that we called the global test pair in experiment 2. 


\subsection{Method}

4.1.1 Participants. Twenty students at the University of Nijmegen participated in this experiment. All had normal or corrected-to-normal vision. Participants received either a small payment or partial course credit.

4.1.2 Stimuli. In this experiment, only the first set from experiment 2 was used, so there was only one occlusion prime (see figure 5). However, this time multiple completions were used that followed the pattern (ie the sequence of concave and convex angles) of the visible part of the partly occluded shape, and one local completion. We have created three variations where protrusions were shorter (or were even absent in the local completion) and three completions where protrusions were longer than the global completion that was used in the previous experiment. The global completion of the previous experiment was used here as well. Figure 8 shows the seven different completions, labeled $A$ to $G$, starting with the completion without a protrusion. As can be seen, completion $A$ is the 'local' completion used in experiment 1 , while completion $D$ is the 'global' completion. These different completions were also used as foreground primes to be able to compare the results of the occlusion prime to the effect of the congruent foreground primes. In total, there were eight different primes. The no-prime display consisted of a dot in the middle of the screen, like in experiment 1.

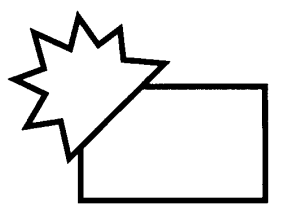

A

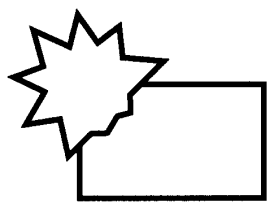

B

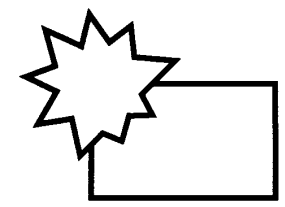

C

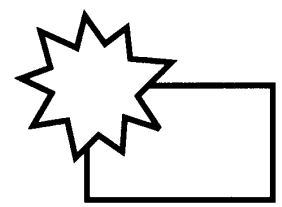

$\mathrm{D}$
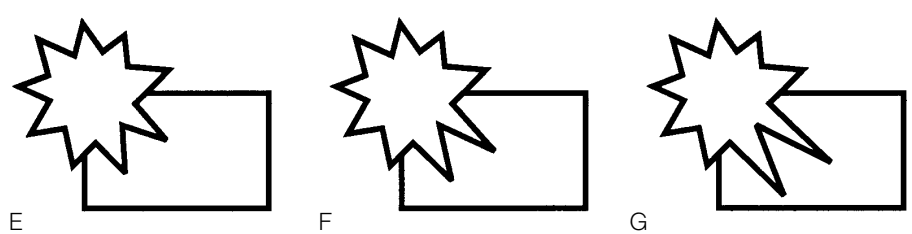

G

Figure 8. The seven foreground primes for experiment 3. In prime B protrusions are added in the completed part and the length of these protrusions increases from left to right.

Similar to the previous experiment, there were matching and non-matching test pairs. To prevent participants from having to search too long for differences, only non-matching test pairs were used that were clearly different from each other. These non-matching test pairs were formed by pairing one completion with another completion that differed from each other by three or four steps. So, the possible non-matching combinations were $A D, A E, B E, B F, C F, C G$, and $D G$. The left-right position was varied for every pair making a total of fourteen non-matching pairs and seven matching pairs.

4.1.3 Procedure. The procedure was the same as that in experiment 2 . In the present experiment, foreground primes either preceded matching test pairs with which the foreground primes were congruent (eg after foreground prime $A$ only test pair AA was shown), or non-matching test pairs that contained one of the shapes with the completion that was already in the foreground prime (eg after foreground prime A only test pair $A D$ or $A E$ were presented). The blank screen between the fixation cross and the prime lasted for $150 \mathrm{~ms}$. There was a total of 504 experimental trials.

\subsection{Results}

Similar to experiment 2, the correctly answered matching test pairs (93.1\% of all trials) were analysed. Figure 9 depicts the mean prime effect for the foreground and occlusion 
primes as a function of different test pairs. First, a paired-samples $t$-test was performed to see if the foreground primes significantly affected RTs for congruent test pairs. The findings showed the same prime effect as experiment 1, where foreground primes shortened RTs for congruent test pairs. This was found for all completions [PE (A|A): $t=-5.851, \quad p<0.001 ; \quad \mathrm{PE}(\mathrm{B} \mid \mathrm{B}): t=-3.688, p<0.005 ; \quad \mathrm{PE} \quad(\mathrm{C} \mid \mathrm{C}): t=-4.990$, $p<0.001 ; \mathrm{PE}(\mathrm{D} \mid \mathrm{D}): t=-2.951, p<0.001 ; \mathrm{PE}(\mathrm{E} \mid \mathrm{E}): t=-5.440, p<0.001 ; \mathrm{PE}$ $(\mathrm{F} \mid \mathrm{F}): t=-2.972, p<0.001 ; \mathrm{PE}(\mathrm{G} \mid \mathrm{G}): t=-7.225, p<0.001]$. Next, a paired-samples $t$-test was performed to see for which test pairs the RTs were significantly affected by the occlusion prime. Test pairs which were facilitated by the occlusion prime were: test pair $\mathrm{C}(t=4.873, p<0.001)$, D $(t=4.185, p<0.005)$, and $\mathrm{E}(t=2.912, p<0.01)$. Also, the results from these combinations did not significantly differ from the effect of the congruent foreground primes on these test pairs.

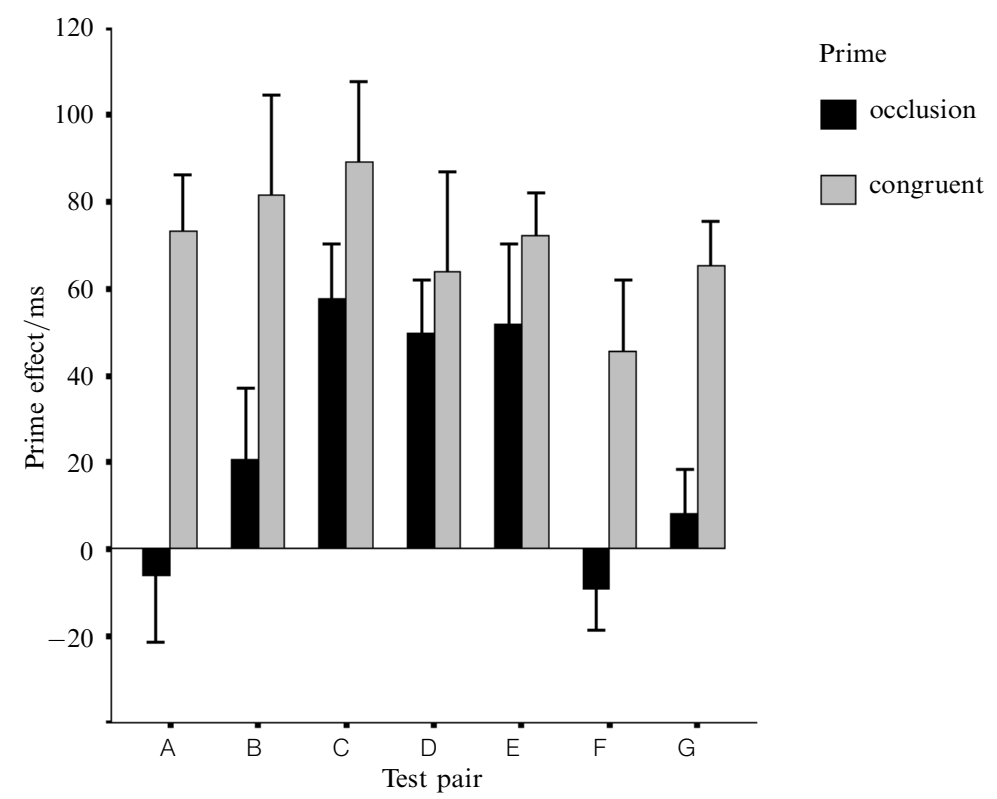

Figure 9. Mean prime-effect as a function of different test pairs for experiment 3. The bars represent mean standard error.

\subsection{Discussion}

Like in the previous experiment, it was found that foreground primes improved performance for test pairs that they were congruent with. This prime effect was found for all the different completions. However, to answer our question regarding the range of plausible completions, we have to look at the effect of the occlusion prime on the different primes. The occlusion prime improved performance for three different test pairs, so multiple completions were primed by the occlusion prime. More specifically, the completions that were formed after seeing the partly occluded prime were the completions that were most similar to the overall pattern of the visible part of the occlusion prime. Again no local completions were primed and the results provide more support for our idea that there are global influences in the completion of partly occluded shapes outside the usual regular stimulus domain. Furthermore, the results from this experiment give us a small peak at the variation in the completion, but it goes beyond the scope of this paper to say something about the exact nature of the variations in the completions. 
For the partly occluded shape used here, the variability of the multiple completions was found in certain metrical aspects of the completed part. Not only did the occlusion prime have an effect on the test pair that we referred to as the global test pair in experiment 2, it also had an effect on the two neighbouring completions. This can tell us something about the variation in the completion process. The fact that the other completions were not influenced by the occlusion prime might suggest that the boundary of the possible variation for plausible completions lies somewhere near completion $C$ and completion E. Completions $\mathrm{C}$ and $\mathrm{E}$ do not necessarily have to be formed in order to be primed, and therefore the precise location of the boundary remains unclear. That is, even if the variation in the completion is smaller than the difference between $C$ and $E$, this variation could still have a prime effect on $C$ and $E$. This effect could be due to the similarity between the variation completion and the completions used here. However, an alternative explanation for the findings is conceivable. There is a possibility that participants simply could not see the difference between completions C and D, and between completions $D$ and $E$. If this is the case, the findings from this experiment cannot be used to make a statement about the degree of variation in the completion process. To control for this alternative explanation, we performed an additional experiment to see whether people can differentiate between the three completions. Again, we used the primed-matching paradigm.

\section{Control experiment}

We want to find out if the prime effect of the occlusion prime for the test pairs $C, D$, and $E$ is caused by some inability of the participants to see the difference between these shapes. One way to investigate if people are able to see the difference between $C, D$, and $E$ is to use the primed-matching paradigm again and look at the effect of foreground primes $C, D$, and $E$ on the test pairs $C, D$, and E. If the primes have an effect on their congruent test pairs but not on the other test pairs, then people are able to see the difference between the different completions. We were mainly interested in the effect of foreground primes $C$ and $E$ on test pairs $C$ and $E$, respectively, and on test pair D. And we were interested in the effect of prime $D$ on test pairs $C, D$, and $E$, so foreground primes only preceded test pairs they were congruent with and test pairs containing neighbouring completions.

\subsection{Method}

5.1.1 Participants. Twenty students at the University of Nijmegen participated in this experiment. All had normal or corrected-to-normal vision. Participants received either a small payment or partial course credit.

5.1.2 Stimuli. In this experiment only a subset of the stimuli of experiment 3 (figure 8) was used. To avoid a frequency difference between the occurrence of the relevant test pairs $C, D$, and $E$, because of the fact that test pairs were always shown after their neighbouring primes, foreground primes B to $F$ were used. All test stimuli were used, but we were only interested in the results for test pairs C, D, and E.

5.1.3 Procedure. The procedure was similar to that used in the previous experiments, except that now foreground primes always preceded test pairs containing congruent completions and test pairs containing neighbouring completions (eg after completed prime $D$, only test pairs $C C, D D$, or $E E$, or a non-matching pair with one of the shapes was presented). There was a total of 352 experimental trials.

\subsection{Results}

Matching test pairs correctly answered (94.0\% of all trials) were analysed. First, a paired-samples $t$-test was performed to see if the foreground primes significantly facilitated performance for congruent test pairs. The findings showed the same 
prime effect as in experiment 1, where foreground primes shortened RTs for congruent test pairs. This was found for all completions [PE $(\mathrm{C} \mid \mathrm{C}): t=3.172, p<0.01$; PE (D|D): $t=3.12, p<0.01$; PE (E|E): $t=4.062, p<0.005]$. Next, we analysed the effects of the foreground primes on the different test pairs. There was a prime effect of prime $\mathrm{D}$ on test pair $\mathrm{E}[\mathrm{PE}(\mathrm{D} \mid \mathrm{E}): t=2.264, p<0.05]$ but not on test pair $\mathrm{C}$ [PE (D|C): $t=-0.042, p=0.976]$. Also, test pair $\mathrm{D}$ was not influenced by prime $\mathrm{C}$ [PE (C|D): $t=-1.045, p=0.309]$, nor by prime $\mathrm{E}$ [PE (E|D): $t=-1.438, p=0.167]$.

\subsection{Discussion}

The findings showed the same prime effect as in previous experiments, where foreground primes speed up congruent test pairs. Furthermore, foreground prime $D$ facilitated the response for test pair $E$, but there was no prime effect of foreground prime $D$ on test pair C. In addition, there was no prime effect from foreground primes $C$ and $E$ on test pair D. From this it can be concluded that participants could clearly see the difference between completions $\mathrm{C}$ and $\mathrm{D}$, and between completions $\mathrm{D}$ and $\mathrm{E}$. Thus, the finding in experiment 2 regarding the prime effect of the occlusion prime on completions $C$ and $E$ cannot be explained by some inability of the visual system to see the difference between the completions.

Of course, the exact nature of the prime effect is not clear, but looking at the different completions that are still primed might give an indication regarding the nature of the variation in the completion process. Nevertheless, it seems reasonable to assume that the variation in the completion will be between $C$ and $E$. The fact that foreground prime $D$ does have a facilitating effect on test pair $E$ has another implication for experiment 3. The prime effect of the occlusion prime on completion $E$ may lie not only in the formation by the completion process, but this effect could also partly have been caused by a more indirect priming mechanism. Completion $E$ does not have to be formed itself, but it could have been primed by another completion that was formed. This other completion might have primed completion $E$ owing to the similarity between the formed completion and E. The important finding here, however, is that the prime effect of the occlusion prime on completions $C$ and $E$, found in experiment 3 , is not due to some inability to see the difference between the different completions.

\section{General discussion}

The present experiments were set up to investigate the susceptibility of the visual system to global processes in the visual completion of quasi-regular shapes. In experiment 1 , we found that participants spontaneously complete partly occluded quasi-regular shapes in a global way. In experiment 2 this evidence for global completions was replicated with another task: the primed-matching paradigm. Here, we found that global completions are facilitated more by an occlusion prime than are local completions. Subsequently, experiment 3 gave a hint about the flexibility of the completions, because it showed that a range of completions was primed after an occlusion prime. A control experiment showed that this finding cannot be explained by some inability of the visual system to see the difference between the various completions. In conclusion, the main result from the experiments described here is that evidence is provided for the robustness of global influences. It has become clear that global influences can dominate the completion process even outside the domain of regular shapes.

An important point of discussion is whether the visual system always generates possible completions for partly occluded shapes, or whether it may just conclude that there is a part of the shape behind an occluder. Particularly for complex shapes or objects the latter option seems more conceivable, because the range of possibilities is larger for complex shapes than for simple objects. Obviously, a partly occluded square has one single compelling solution whereas the back of an (arbitrary) complex object 
has many plausible solutions. The more plausible completions exist, the lower the activation for each single completion could be (van Lier 1999). In the latter case, a larger cognitive effort is needed for selecting a specific choice as a solution. However, the range of plausible solutions out of which this choice is made might still be perceptually defined. The results of our primed-matching experiments indeed seem to be evidence for this claim, since we find priming effects for perceptual completions even for the more complex shapes that we used.

Another important point of discussion is which theories could handle these results. It has been noted that, of the theories that were mentioned in the Introduction, no theory would have predicted these 'global' findings. Obviously, local theories would have predicted local completions and global theories would have problems in predicting these global influences to dominate in the completion process when there is no regularity present in the partly occluded shape. Therefore they would also have predicted the same completions as the local theories. However, these global theories are eligible for modifications such that they also incorporate (global) completion of quasi-regular shapes.

We will try to illustrate this using the structural information theory (SIT) initiated by Leeuwenberg $(1969,1971)$. However, it is not our intention to make strong claims about the actual workings of the visual system. Instead, SIT is a representational theory that can be used to predict completions on the basis of regularities. We will provide a short description of the application of SIT and how it could be modified to account for global completions for partly occluded shapes outside the domain of regular shapes. In SIT, shapes (or objects) are described by a sequence of symbols. This so-called semantic mapping is done by assigning symbols to characteristics of a shape or an object that are metrically identical. For example, a square is composed of four equal lines (1) and four equal angles (a). So, by tracing the square it can be described by the symbol series 'lalalala'. Now, this array of labels can be minimised by extracting regularity and describing this regularity, in terms of iteration, symmetry, and alternation (van der Helm et al 1992). In our example we could do this by describing the iteration of alternating lines and angles in the simplest code $4 *(1 \mathrm{a})$. The complexity of these codes is captured in the information load (I-load) which is 3 in the present example, as determined by the number of parameters in the code. [For the exact details concerning the way in which this code is brought about, and the calculation of the I-load we refer to van der Helm et al (1992).] In the case of amodal completion, these steps are taken for the differently completed shapes, each giving rise to a different simplest code. Out of these different simplest codes, the interpretation with the smallest $I$-load is predicted to be the preferred completion.

As mentioned earlier, only characteristics of a shape that are identical are to be labeled by the same symbol. Characteristics of a shape do not necessarily have to be metrically identical to describe certain regularities (see also van Lier 1999). More specifically, the labels in the series of symbols may refer to some a priori categorised properties, instead of metrical identities. The concave and convex angles that we already used in the description of the stimuli in our experiments can be considered as examples of such a priori properties. For the different completions for the shapes of experiment 2 codes based on such a priori properties for the component and their $I$-loads can be found in table 2, which are smallest for the global completions. Thus, on the basis of these codes, global completions would be predicted.

Although this 'fuzzy' encoding would result in appropriate classifications, future research should be clearer in the specification of the a priori categories, which is not an easy task. One problem is that the categorisations are very much tied to the shape itself. There are no absolute boundaries for the categorisations, so these have to be artificially fixed in some way. For example, in the stimuli that we used, one could 
Table 2. Codes and $I$-loads for the occluded shapes and their completions for all sets from experiment 2 (following the suggestion for a priori categories, see text). 1 stands for a line, $v$ stands for a convex angle, and c stands for a concave angle; $\mathrm{S}$ stands for a symmetry.

\begin{tabular}{lllll}
\hline Shape set & Global completion & \multicolumn{2}{l}{ Local completion } & \\
\cline { 2 - 4 } & code & $I$-Load & code & -Load \\
\hline 1 & $9^{*}(<1>/<\mathrm{c}><\mathrm{v}>)$ & 4 & $(1)(\mathrm{v}) 6^{*}(<1>/<\mathrm{c}><\mathrm{v}>)$ & 6 \\
2 & $6^{*}((\mathrm{v}) \mathrm{S}[(1)(\mathrm{c}),(1)])$ & 5 & $(\mathrm{v})(\mathrm{l}) 4^{*}((\mathrm{v}) \mathrm{S}[(1)(\mathrm{c}),(1)])$ & 7 \\
3 & $7^{*}(\mathrm{~S}[(\mathrm{v})(1)(\mathrm{c}),(1)](1))$ & 6 & $(\mathrm{v})(\mathrm{l}) 5^{*}(\mathrm{~S}[(\mathrm{v})(1)(\mathrm{c}),(1)])$ & 7
\end{tabular}

make a differentiation between 'short' and 'long' lines. If these 'short' lines were part of another shape that contained even shorter lines, these same lines could be classified as 'long' lines because the internal reference has changed. Another problem that has to be dealt with in future research, besides these internal factors of a shape, is the influence of surrounding shapes and objects. That is, in our natural world other shapes that are present in the visual image will also influence the interpretation of shape categories. So, besides the shape-dependence, there is also a contextual dependence. Specific assumptions regarding the nature of the categorical properties would go beyond the scope of this study which was set up to advocate the flexibility of the completion process with regard to global influences. An implication of a more flexible approach is that it helps us in accounting for a certain variability in a broader range of partly occluded shapes outside the domain of regular shapes. Exploring the boundaries of the categorical properties might also give more insight into the possible variations in completions. Every model that tries to predict completions on the basis of these figural properties is faced with the problem of capturing the necessary characteristics that constitute quasi-regularities.

No matter how such models develop, the experiments reported here showed that our visual system is still sensitive to these quasi-regularities even in the case of completions.

\section{Conclusion}

We conclude that global influences can dominate the completion process even outside the domain of partly occluded regular shapes.

Acknowledgments. TdW was funded by the Netherlands Organisation of Scientific Research (NWO) and RvL received a grant from the Royal Netherlands Academy of Arts and Sciences. Part of this study was presented at the first annual meeting of the Vision Sciences Society and at the 24th meeting of the European Conference on Visual Perception. The authors would like to thank Peter van der Helm, Arno Koning, Maan Leeuwenberg, Johan Wagemans, and three anonymous reviewers for their helpful comments on an earlier version of this manuscript.

\section{References}

Beller H, 1971 "Priming: effects of advance information on matching" Journal of Experimental Psychology $87176-182$

Boselie F, 1988 "Local versus global minima in visual pattern completion" Perception \& Psychophysics $\mathbf{4 3} 431-445$

Boselie F, 1994 "Local and global factors in visual occlusion" Perception 23 517-528

Boselie F, Wouterlood D, 1992 "A critical discussion of Kellman and Shipley's (1991) theory of occlusion phenomena" Psychological Research 54278 -285

Buffart H, Leeuwenberg E, Restle F, 1981 "Coding theory of visual pattern completion" Journal of Experimental Psychology: Human Perception and Performance $7241-274$

Buffart H, Leeuwenberg E, Restle F, 1983 "Analysis of ambiguity in visual pattern completion" Journal of Experimental Psychology: Human Perception and Performance $9980-1000$

Helm P van der, Lier R van, Leeuwenberg E, 1992 "Serial pattern complexity: irregularity and hierarchy" Perception $21517-544$

Hochberg J, McAlister E, 1953 "A quantitative approach to figural 'goodness'” Journal of Experimental Psychology $46361-364$ 
Kanizsa G, 1975 "The role of regularity in perceptual organization", in Studies in Perception Ed. F d'Arcais (Florence: Martello) pp 48-66

Kanizsa G, 1979 Organization in Vision. Essays on Gestalt Perception (New York: Praeger)

Kanizsa G, 1985 "Seeing and thinking" Acta Psychologica 5923 -33

Kellman P J, Shipley T F, 1991 "A theory of visual interpolation in object perception" Cognitive Psychology $23141-221$

Koffka K, 1962 Principles of Gestalt Psychology (London: Routledge \& Kegan Paul)

Leeuwenberg E, 1969 "Quantitative specification of information in sequential patterns" Psychological Review $76216-220$

Leeuwenberg E, 1971 "A perceptual coding language for visual and auditory patterns" Psychological Review $76307-349$

Lier R van, 1999 "Investigating global effects in visual occlusion: from a partly occluded square to the back of a tree-trunk" Acta Psychologica 102 203-220

Lier R van, Helm P van der, Leeuwenberg E, 1994 "Integrating global and local aspects of visual occlusion" Perception 23 883-903

Lier R van, Helm P van der, Leeuwenberg E, 1995b "Competing global and local completions in visual occlusion" Journal of Experimental Psychology: Human Perception and Performance $21571-583$

Lier R van, Leeuwenberg E, Helm P van der, 1995a "Multiple completions primed by occlusion patterns" Perception $24727-740$

Lier R van, Wagemans J, 1999 "From images to objects: global and local completions of selfoccluded parts" Journal of Experimental Psychology: Human Perception and Performance $\mathbf{2 5}$ $1721-1741$

Rock I, 1983 The Logic of Perception (Cambridge, MA: MIT Press)

Rubin N, 2001 "The role of junctions in surface completion and contour matching" Perception $30339-366$

Sekuler A B, 1994 "Local and global minima in visual completion: effects of symmetry and orientation" Perception $23529-545$

Sekuler A B, Palmer S E, 1992 "Perception of partly occluded objects: a microgenetic analysis" Journal of Experimental Psychology: General $12195-111$

Sekuler A B, Palmer S E, Flynn C, 1994 "Local and global processes in visual completion" Psychological Science $5260-267$

Shipley T F, Kellman P J, 1992 "Perception of partly occluded objects and illusory figures: evidence for an identity hypothesis" Journal of Experimental Psychology: Human Perception and Performance 18 106-120

Wertheimer M, 1923 "Untersuchungen zur Lehre von der Gestalt" Psychologische Forschung 4 $301-350$

Wouterlood D, Boselie F, 1992 "A good-continuation model of some occlusion phenomena" Psychological Research $54267-277$

Yin C, Kellman P J, Shipley T F, 1997 "Surface completion complements boundary interpolation in the visual integration of partly occluded objects" Perception $261459-1479$ 